\title{
Two Numerical Methods (RO (MSuM) and RO (SuMSu)) for Triple Integrals with for Continuous Functions
}

\author{
Safaa M. Aljassas*, Dhuha Abdulameer Kadhim, Eman Yahea Habeeb \\ Department of Mathematics, College of Education for Girls, University of Kufa, Najaf 54002, Iraq
}

Corresponding Author Email: aljassas.safaa@yahoo.com

https://doi.org/10.18280/mmep.080417

Received: 27 September 2020

Accepted: 12 April 2021

\section{Keywords:}

triple Integrals, continuous functions, Mid-Point

Rule, suggested method, Romberg accelerating

\begin{abstract}
The main goal of this research is to calculate a triple integral included continuous integrands numerically by two composite rules. The first rule is the Mid-point method on the third dimension $\mathrm{Z}$ and the first dimension $\mathrm{X}$ with a suggested method on the second dimension $\mathrm{Y}$, that is denoted by $M S u M$. The second rule is the suggested method on the third dimension $\mathrm{Z}$ and the first dimension $\mathrm{X}$ with a Mid-point method on the second dimension Y, that is denoted by $S u M S u$. The number of partial intervals is equals on the three dimensions. The study represented two theorems with the proofs to get such rules and the correction terms (the error terms) for each of rule. Moreover, to accelerate convergence and get better results, Romberg acceleration is used with both rules. These rules recalled by $R O(M S u M)$ and $R O(S u M S u)$ respectively such that the obtained results were high accuracy by relatively few partial intervals and shorter times.
\end{abstract}

\section{INTRODUCTION}

The triple integrals are important in finding volumes, intermediate centers, and inertia torque of volumes. For example the size between the parabola, the cylinder, the volume inside the cylinder specified from the top of the sphere and from the bottom of the plane, calculating the average center of the size falling in and over the plane and below the plane. In addition, its important in Find blocks of variable density such as a thin wire or a thin plate of metal [1]. Many investigators studied the field of triple integrals, one of them Dheyaa [2]. He used two rules of Newton-Cotes rules (the Mid- point and Simpson methods) with Romberg acceleration to configure four composite numerical methods which are $R M R M(R S), R M R M(R M), R M R S(R M)$ and $R M R S(R S)$. In terms of time, accuracy and number of sub-intervals, the said study claimed that the composite method is the best method for the calculation of triple integrals involving continuous integration.

In another regard, and using Romberg 's acceleration of the conclusion values obtained from the Midpoint method applied to Eghaar [3] proposed a numerical method for the calculation of triple integrals. Furthermore, the study showed that the obtained results was a good in terms of accuracy.

Muosa et al. [4] studied a numerical method for calculate triple integrals by using Aitken's Acceleration of the conclusion values which obtained from applied Mid-point rule, on the dimension $\mathrm{z}$ and Simpson's rule on the two dimensions $\mathrm{x}$ and $\mathrm{y}$, symbolized by. Subsequently, they were given good results.

There are many researchers who introduced numerical methods that mixed between the Mid- point. They used Romberg acceleration or Aitken's Acceleration to get good rustles in terms of accuracy and speed depending on the number of sub- intervals and times [5-7]. Sarada and Nagarajan [8] employed the generalized Gaussian Quadrature to calculate triple integrals and obtained a great result. Aljassas [9] employed Romberg acceleration with Mid-point rule on the three dimensions to calculate riple integrals with continuous functions. In addition, many authors introduced a new method to increase the accuracy of numerical integrals results [10-13].

In this paper, we study two theorems contain their proofs in order find new numerical methods and their correction terms in order to calculate the approximate values for the triple integrals that continuous integrands. These two methods are derived from the application of Romberg acceleration besides the two composite rules, (first from the Mid-point method on the third and the first dimensions with a suggested method on the second dimension) and (second from the suggested method on the third and the first dimensions with a Mid-point method on the second dimension) where the number of divisions on these three dimensions is equal.

\section{MID-POINT METHOD}

Assume integral $I$ is defined by:

$$
I=\int_{a}^{b} h(\alpha) d \alpha=M(t)+\delta_{M}(t)+R_{M}
$$

where, $M(t)$ is the Mid-point method, $\delta_{M}(t)$ be the correction terms series of $M(t)$ and $R_{M}$ is the remainder from truncation terms $\delta_{M}(t)$ after using certain terms of $\delta_{M}(t)$.

The general formula of the Mid-point method [14] is:

$$
M(t)=t \sum_{i=1}^{w}\left(a+\frac{(2 i-1)}{2} t\right)
$$


The correction terms series of Mid- point method of continuous integrands using the mean value theorem will be:

$$
\begin{aligned}
\delta_{M}(t) & =\frac{(b-a)}{6} t^{2} h^{(2)}\left(\eta_{1}\right)-\frac{7(b-a)}{360} t^{4} h^{(4)}\left(\eta_{2}\right)+ \\
& +\frac{31(b-a)}{15120} t^{6} h^{(6)}\left(\eta_{3}\right)-\cdots
\end{aligned}
$$

Such that $i=1,2,3, \ldots, \eta_{i} \in(a, b)[3]$.

$$
R_{M}(t)=\frac{w}{2 R} B_{2 R} t^{2 R+1} h^{2 R}(\lambda)
$$

where, $a<\lambda<b$ and $B_{2 R}$ is Bernoulli number Dheyaa [2] (The highest derivative present within the correction set is of the order $(2 R+3)$, so when $t$ is fixed, the remainder in general will result in zero with an increase in $R$, but if it is $R$ constant then here is a small value for $t$ that makes us neglect $R_{M}$ to obtain the required accuracy).

\section{SUGGESTED METHOD (SU)}

The Proposed method that characterized by Muhammad et al. [15], derived from the Trapezoidal and Mid-point methods which are used to calculate the single integrals and gave fine outcomes in accuracy and speed of approach better than the Mid-point and Trapezoidal methods.

The general formula of this method is:

$$
\begin{aligned}
& S u(t)=\frac{t}{4}[h(a)+h(b)+2 h(a+(w-0.5) t)+ \\
& \left.2 \sum_{i=1}^{w-1}(h(a+(i-0.5) t)+h(a+i t))\right]
\end{aligned}
$$

And the correction terms series are:

$$
\begin{gathered}
\delta_{S u}(t)=\frac{1}{24} t^{2}\left(h_{w}^{\prime}-h_{0}^{\prime}\right)-\frac{5}{1440} t^{4}\left(h_{w}^{(3)}-h_{0}^{(3)}\right)+ \\
+\frac{61}{60480} t^{6}\left(h_{w}^{(5)}-h_{0}^{(5)}\right)-\cdots
\end{gathered}
$$

Using the Mean value theorem calculus for (6), we have

$$
\begin{aligned}
& \delta_{S u}(t)=\frac{(b-a)}{24} t^{2} h^{(2)}\left(\mu_{1}\right)-\frac{5(b-a)}{1440} t^{4} h^{(4)}\left(\mu_{2}\right)+ \\
& +\frac{61(b-a)}{60480} t^{6} h^{(6)}\left(\mu_{3}\right)-\cdots
\end{aligned}
$$

\section{ROMBERG ACCELERATING}

Assuming the following integral

$$
I=\int_{a}^{b} h(\alpha) d \alpha
$$

If we calculate the value of the integral $I$ for two different approximate values of $t$ which are $t_{2}, t_{1}$, suppose that one of these values is $\lambda_{1}$ and the second is $\lambda_{2}$ respectively which are approximated values by one of Newton-coats methods. So the integral I will be:

$$
\begin{gathered}
I=\int_{a}^{b} h(\alpha) d \alpha=\lambda_{1}+\sum_{j=R}^{\infty} A_{j} t_{1}^{j} \\
I=\int_{a}^{b} h(\alpha) d \alpha=\lambda_{2}+\sum_{j=R}^{\infty} A_{j} t_{2}^{j}
\end{gathered}
$$

where, $\lambda_{1}, \lambda_{2}$ two approximate values for integral by using one of Newton-Coates methods, $I$ is the exact -integration $\sum_{j=R}^{\infty} A_{j} t_{1}^{j}$ are the correction terms.

$$
\begin{aligned}
& I=\int_{a}^{b} h(\alpha) d \alpha=\lambda_{1}+A_{R} t_{1}^{j}+\sum_{j=R+1}^{\infty} A_{j} t_{1}^{j} \\
& I=\int_{a}^{b} h(\alpha) d \alpha=\lambda_{2}+A_{R} t_{2}^{j}+\sum_{j=R+1}^{\infty} A_{j} t_{2}^{j}
\end{aligned}
$$

Multiplying the first formula by $t_{2}^{j}$ and the second by $t_{1}^{j}$ we get:

$$
\begin{aligned}
& t_{2}^{j} I=t_{2}^{j} \int_{a}^{b} h(\alpha) d \alpha=\lambda_{1} t_{2}^{j}+A_{R} t_{1}^{j} t_{2}^{j}+\sum_{j=R+1}^{\infty} A_{j} t_{1}^{j} t_{2}^{j} \\
& t_{1}^{j} I=t_{2}^{j} \int_{a}^{b} h(\alpha) d \alpha=\lambda_{2} t_{1}^{j}+A_{R} t_{2}^{j} t_{1}^{j}+\sum_{j=R+1}^{\infty} A_{j} t_{2}^{j} t_{1}^{j}
\end{aligned}
$$

Subtract 11 from 12 we get:

$$
\begin{aligned}
& I=\int_{a}^{b} h(\alpha) d x=\frac{t_{1}^{R} \lambda_{2}-t_{2}^{R} \lambda_{1}}{t_{1}^{R}-t_{2}^{R}} \\
& +\sum_{j=R+1}^{\infty} A_{j} \frac{t_{1}^{R} t_{2}^{j}-t_{2}^{R} t_{1}^{j}}{t_{1}^{R}-t_{2}^{R}}
\end{aligned}
$$

Now, let $t_{2}=2 t_{1}$, Eq. (13)

$$
\begin{aligned}
I & =\int_{a}^{b} h(\alpha) d x=\frac{2^{R} t_{2}{ }^{R} \lambda_{2}-t_{2}^{R} \lambda_{1}}{2^{R} t_{2}{ }^{R}-t_{2}^{R}}+ \\
& +\sum_{j=R+1}^{\infty} A_{j} \frac{2^{R} t_{2}^{R} t_{2}^{j}-2^{j} t_{2}^{R} t_{2}^{j}}{2^{R} t_{2}^{R}-t_{2}^{R}}
\end{aligned}
$$


Reducing equal quantities, we get

$$
I=\frac{2^{R} \lambda_{2}-\lambda_{1}}{2^{R}-1}+\sum_{j=R+1}^{\infty} \frac{2^{R}-2^{j}}{2^{R}-1} A_{j} t^{j} .
$$

Thus, the formula of Romberg accelerating will be:

$$
I \cong \frac{2^{R} \lambda_{2}-\lambda_{1}}{2^{R}-1}
$$

$\sum_{j=R+1}^{\infty} \frac{2^{R}-2^{j}}{2^{R}-1} A_{j} t^{j}$ is cutting error when $R$ is the power of $\mathrm{t}$ in the correction terms [16].

We took $t_{2}=2 t_{1}$ meaning $t_{2}$ would be double $t_{1}$ because this division achieves better results with Romberg Accelerating as shown in the examples.

Other divisions were taken, such as $t_{2}=3 t_{1}, t_{2}=$ $4 t_{1}, t_{2}=5 t_{1}, \ldots$ the results were not as good as the results we obtained when using $t_{2}=2 t_{1}$.

\section{THE TWO NUMERICAL METHODS MSUM, SUMSU AND THE CORRECTION TERMS}

\subsection{Numerical method MSuM}

Theorem(1): If $\mathrm{h}(\alpha, \kappa, \rho)$ differentiable and continuous function at any point of the region $D=[a, b] \times[c, d] \times[e, f]$ then the approximated value of integral $I=$ $\iint_{D} \int h(\alpha, \kappa, \rho) d \alpha d \kappa d \rho$ can be determined by:

$$
\begin{gathered}
M S u M=\frac{t^{3}}{4} \sum_{\ell=1}^{w} \sum_{i=1}^{w}\left[h\left(\alpha_{i}, c, \rho_{\ell}\right)+2 h\left(\alpha_{i}, c\right.\right. \\
\left.+(w-0.5) t, \rho_{\ell}\right) \\
\left.+2 \sum_{j=1}^{w-1}\left(h\left(\alpha_{i}, c+(j-0.5) t, \rho_{\ell}\right)+h\left(\alpha_{i}, c+j t, \rho_{\ell}\right)\right)\right] \\
\alpha_{i}=a+\left(\frac{2 i-1}{2}\right) t, i=1,2, \ldots, w \\
\rho_{\ell}=e+\frac{(2 \ell-1)}{2} \mathrm{t}, \quad \ell=1,2, \ldots, w
\end{gathered}
$$

Also, the correction terms is

$$
I-M S u M(t)=\gamma_{1} t^{2}+\gamma_{2} t^{4}+\gamma_{3} t^{6}+\cdots
$$

when $\gamma_{1}, \gamma_{2}, \gamma_{3}, \cdots$ are constants.

Proof: We could rewrite the integral $I$ by:

$$
\begin{aligned}
& I=\int_{e}^{f} \int_{c}^{d} \int_{a}^{b} h(\alpha, \kappa, \rho) d \alpha d \kappa d \rho \\
& =\operatorname{MSuM}(t)+\delta_{\text {MSuM }}(t)
\end{aligned}
$$

where, $\operatorname{MSuM}(\mathrm{t})$ : is the approximation value of integral.

Suggested method on the second dimension with a Midpoint method on the third dimension, $\delta_{M S u M}(t)$ are the correction terms that could be added to the values of $\operatorname{MSuM}(t)$, and $\mathrm{t}=\frac{(\mathrm{b}-\mathrm{a})}{\mathrm{w}}=\frac{(\mathrm{d}-\mathrm{c})}{\mathrm{w}_{1}}=\frac{(\mathrm{f}-\mathrm{e})}{\mathrm{w}_{2}}$.

The single integral $\int_{a}^{b} h(\alpha, \kappa, \rho) d \alpha$ can be calculate numerically by Mid-point method on the dimension X (dealing with $y$ and $z$ as constants), so:

$$
\begin{aligned}
& \int_{a}^{b} h(\alpha, \kappa, \rho) d \alpha=t \sum_{i=1}^{w} h\left(a+\frac{(2 i-1)}{2} t, \kappa, \rho\right) \\
& +\frac{(b-a)}{6} t^{2} h_{x^{2}}\left(\varphi_{1}, \kappa, \rho\right) \\
& -\frac{7(b-a)}{360} t^{4} h_{x^{4}}\left(\varphi_{2}, \kappa, \rho\right) \\
& +\frac{31(b-a)}{15120} t^{6} h_{x^{6}}\left(\varphi_{3}, \kappa, \rho\right)-\cdots
\end{aligned}
$$

Integrate (18) numerically on the interval [c,d] by using suggested method on the dimension $\kappa$, we obtain:

$$
\begin{aligned}
& \int_{c}^{d} \int_{a}^{b} h(\alpha, \kappa, \rho) d \alpha d \kappa \\
& =\frac{t^{2}}{4} \sum_{i=1}^{w}\left[h\left(\alpha_{i}, c, \rho\right)+h\left(\alpha_{i}, d, \rho\right)\right. \\
& \quad+2 h\left(\alpha_{i}, c+(w-0.5) t, \rho\right) \\
& \left.\left.\quad+2 \sum_{j=1}^{w-1}\left(h\left(\alpha_{i}, c \quad j-0.5\right) t, \rho\right)+h\left(\alpha_{i}, c+j t, \rho\right)\right)\right]+ \\
& +\int_{c}^{d}\left[\frac{(b-a)}{6} t^{2} h_{\alpha^{2}}\left(\varphi_{1}, \kappa, \rho\right)\right. \\
& -\frac{7(b-a)}{360} t^{4} h_{\alpha^{4}}\left(\varphi_{2}, \kappa, \rho\right)+ \\
& \left.+\frac{31(b-a)}{15120} t^{6} h_{\alpha^{6}}\left(\varphi_{3}, \kappa, \rho\right)-\ldots\right] d \kappa \\
& +t \sum_{i=1}^{w}\left[\frac{(d-c)}{24} t^{2} h_{\kappa^{2}}\left(\alpha_{i}, \phi_{1 i}, \rho\right)\right. \\
& +\frac{5(d-c)}{1440} t^{4} h_{\kappa^{4}}\left(\alpha_{i}, \phi_{2 i}, \rho\right)+ \\
& \left.+\frac{61(d-c)}{60480} t^{6} h_{\kappa^{6}}\left(\alpha_{i}, \phi_{3 i}, \rho\right)-\ldots\right]
\end{aligned}
$$

Again integrate formula (19) numerically on the interval [e,f] using Mid-point method on the dimension $\mathrm{z}$, we have the following formulas:

$$
\begin{aligned}
& \text { 1) } \int_{e}^{f} h\left(\alpha_{i}, c, \rho\right) d \rho=t \sum_{\ell=1}^{w} h\left(\alpha_{i}, c, \rho_{\ell}\right) \\
& +\left[\frac{(f-e)}{6} t^{2} h_{\alpha^{2}}\left(\alpha_{i}, c, \beta_{1 i}\right)-\frac{7(f-e)}{360} t^{4} h_{\alpha^{4}}\left(\alpha_{i}, c, \beta_{2 i}\right)\right. \\
& \left.+\frac{31(f-e)}{15120} t^{6} h_{\alpha^{6}}\left(\alpha_{i}, c, \beta_{3 i}\right)-\cdots\right]
\end{aligned}
$$


$2) \int_{e}^{f} h\left(\alpha_{i}, d, \rho\right) d \rho=t \sum_{j=1}^{w} h\left(\alpha_{i}, d, \rho_{j}\right)$

$+\left[\frac{(f-e)}{6} t^{2} h_{\alpha^{2}}\left(\alpha_{i}, d, \beta_{1 i}\right)\right.$

$-\frac{7(f-e)}{360} t^{4} h_{\alpha^{4}}\left(\alpha_{i}, d, \beta_{2 i}\right)$

$\left.+\frac{31(f-e)}{15120} t^{6} h_{\alpha^{6}}\left(\alpha_{i}, d, \beta_{3 i}\right)-\cdots\right]$

$$
\begin{aligned}
& 3) \int_{e}^{f}=t \sum_{\ell=1}^{f} h\left(\alpha_{i}, c+(w-0.5) t, \rho\right) d \rho \\
& +\left[\frac{(f-e)}{6} t^{2} h_{\alpha^{2}}\left(\alpha_{i}, c+(w-0.5) t, \beta_{1 i}\right)\right. \\
& -\frac{7(f-e)}{360} t^{4} h_{\alpha^{4}}\left(\alpha_{i}, c+(w-0.5) t, \beta_{2 i}\right) \\
& \left.+\frac{31(f-e)}{15120} t^{6} h_{\alpha^{6}}\left(\alpha_{i}, c+(w-0.5) t, \beta_{3 i}\right)-\cdots\right]
\end{aligned}
$$

${ }_{f} h\left(\alpha_{i}, c+(j-0.5) t, \rho\right) d \rho$

4)

$$
\begin{aligned}
4) \int_{e} & =t \sum_{\ell=1}^{w} h\left(\alpha_{i}, c+(j-0.5) t, \rho_{\ell}\right) \\
& +\left[\frac{(f-e)}{6} t^{2} h_{\alpha^{2}}\left(\alpha_{i}, c+(j-0.5) t, \beta_{1 i j}\right)\right. \\
& -\frac{7(f-e)}{360} t^{4} h_{\alpha^{4}}\left(\alpha_{i}, c+(j-0.5) t, \beta_{2 i j}\right) \\
& \left.+\frac{31(f-e)}{15120} t^{6} h_{\alpha^{6}}\left(\alpha_{i}, c+(j-0.5) t, \beta_{3 i j}\right)-\cdots\right]
\end{aligned}
$$$$
\text { 5) } \int_{e}^{f} h\left(\alpha_{i}, c+j t, \rho\right) d \rho=t \sum_{\ell=1}^{w} h\left(\alpha_{i}, c+j t, \rho_{\ell}\right)
$$$$
+\left[\frac{(f-e)}{6} t^{2} h_{\alpha^{2}}\left(\alpha_{i}, c+j t, \beta_{1 i j}\right)\right.
$$$$
-\frac{7(f-e)}{360} t^{4} h_{\alpha^{4}}\left(\alpha_{i}, c+j t, \beta_{2 i j}\right)
$$$$
\left.+\frac{31(f-e)}{15120} t^{6} h_{\alpha^{6}}\left(\alpha_{i}, c+j t, \beta_{3 i j}\right)\right]-\cdots
$$$$
\text { 6) } \int_{e}^{f} \int_{c}^{d}\left[\frac{7(b-a)}{6} t^{2} h_{\alpha^{2}}\left(\varphi_{1}, \kappa, \rho\right)+\right.
$$$$
\left.+\frac{31(b-a)}{15120} t^{6} h_{\alpha^{6}}\left(\varphi_{3}, \kappa, \rho\right)-\cdots\right] d \kappa d \rho
$$

$$
\begin{aligned}
& \text { 7) } \int_{e}^{f}\left[\frac{(d-c)}{24} t^{2} h_{\kappa^{2}}\left(\alpha_{i}, \phi_{1 i}, \rho\right)\right. \\
& -\frac{5(d-c)}{1440} t^{4} h_{\kappa^{4}}\left(\alpha_{i}, \phi_{2 i}, \rho\right) \\
& \left.+\frac{61(d-c)}{60480} t^{6} h_{\kappa^{6}}\left(\alpha_{i}, \phi_{3 i}, \rho\right)-\ldots\right] d \rho
\end{aligned}
$$

Combining formulas (20), (21), (22), (23), (24), (25) and (26) to get:

$$
\begin{aligned}
& \int_{e}^{f} \int_{c}^{d} \int_{a}^{b} h(\alpha, \kappa, \rho) d \alpha d \kappa d \rho= \\
& \frac{t^{3}}{4} \sum_{\ell=1}^{w} \sum_{i=1}^{w}\left[h\left(\alpha_{i}, c, \rho_{\ell}\right)+h\left(\alpha_{i}, d, \rho_{\ell}\right)\right. \\
& +2 h\left(\alpha_{i}, c+(w-0.5) t, \rho_{\ell}\right) \\
& +2 \sum_{j=1}^{w-1}\left(h\left(\alpha_{i}, c+(j-0.5) t, \rho_{\ell}\right)\right. \\
& \left.\left.+h\left(\alpha_{i}, c+p t, \rho_{\ell}\right)\right)\right] \\
& +\int_{e}^{f} \int_{c}^{d}\left[+\frac{(b-a)}{6} t^{2} h_{\alpha^{2}}\left(\varphi_{1}, \kappa, \rho\right)\right. \\
& -\frac{7(b-a)}{360} t^{4} h_{\alpha^{4}}\left(\varphi_{2}, \kappa, \rho\right) \\
& \left.+\frac{31(b-a)}{15120} t^{6} h_{\alpha^{6}}\left(\varphi_{3}, \kappa, \rho\right)-\cdots\right] d \kappa d \rho \\
& \begin{array}{c}
+t \sum_{i=1}^{w} \int^{f}\left[\frac{(d-c)}{24} t^{2} h_{\kappa^{2}}\left(\alpha_{i}, \phi_{1 i}, \rho\right)\right. \\
-\frac{5(d-c)}{1440} t^{4} h_{\kappa^{4}}\left(\alpha_{i}, \phi_{2 i}, \rho\right)
\end{array}+ \\
& \left.+\frac{61(d-c)}{60480} t^{6} h_{\kappa^{6}}\left(\alpha_{i}, \phi_{3 i}, \rho\right)-\ldots\right] d \rho+ \\
& \begin{array}{r}
+\frac{t^{2}}{4} \sum_{i=1}^{w}\left[\frac{(f-e)}{6} t^{2} h_{\alpha^{2}}\left(\alpha_{i}, c, \beta_{1 i}\right)\right. \\
-\frac{7(f-e)}{360} t^{4} h_{\alpha^{4}}\left(\alpha_{i}, c, \beta_{2 i}\right)
\end{array} \\
& +\frac{31(f-e)}{15120} t^{6} h_{\alpha^{6}}\left(\alpha_{i}, c, \beta_{3 i}\right)-\cdots \\
& +\frac{(f-e)}{6} t^{2} h_{\alpha^{2}}\left(\alpha_{i}, d, \beta_{1 i}\right) \\
& -\frac{7(f-e)}{360} t^{4} h_{\alpha^{4}}\left(\alpha_{i}, d, \beta_{2 i}\right) \\
& +\frac{31(f-e)}{15120} t^{6} h_{\alpha^{6}}\left(\alpha_{i}, d, \beta_{3 i}\right)-\cdots \\
& +2\left(\frac{(f-e)}{6} t^{2} h_{\alpha^{2}}\left(\alpha_{i}, c+(w-0.5) t, \beta_{1 i}\right)\right. \\
& -\frac{7(f-e)}{360} t^{4} h_{\alpha^{4}}\left(\alpha_{i}, c+(w-0.5) t, \beta_{2 i}\right)
\end{aligned}
$$




$$
\begin{aligned}
& \left.+\frac{31(f-e)}{15120} t^{6} h_{\alpha^{6}}\left(\alpha_{i}, c+(w-0.5) t, \beta_{3 i}\right)-\cdots\right) \\
& +2 \sum_{j=1}^{w-1}\left(\frac{(f-e)}{6} t^{2} h_{\alpha^{2}}\left(\alpha_{i}, c+(j-0.5) t, \beta_{1 i j}\right)-\right. \\
& -\frac{7(f-e)}{360} t^{4} h_{\alpha^{4}}\left(\alpha_{i}, c+(j-0.5) t, \beta_{2 i j}\right)+ \\
& +\frac{31(f-e)}{15120} t^{6} h_{\alpha^{6}}\left(\alpha_{i}, c+(j-0.5) t, \beta_{3 i j}\right)-\cdots+ \\
& +\frac{(f-e)}{6} t^{2} h_{\alpha^{2}}\left(\alpha_{i}, c+j t, \beta_{1 i j}\right)- \\
& -\frac{7(f-e)}{360} t^{4} h_{\alpha^{4}}\left(\alpha_{i}, c+j t, \beta_{2 i j}\right)+ \\
& \left.\left.+\frac{31(f-e)}{15120} t^{6} h_{\alpha^{6}}\left(\alpha_{i}, c+j t, \beta_{3 i j}\right)-\cdots\right)\right]
\end{aligned}
$$

Since $h_{\alpha^{2}}, h_{\alpha^{4}}, h_{\alpha^{6}}, \ldots, h_{\kappa^{2}}, h_{\kappa^{4}}, h_{\kappa^{6}}, \ldots$ and $h_{\rho^{2}}, h_{\rho^{4}}, h_{\rho^{6}}, \ldots$ are continuous in each point in the region $[a, b] \times[c, d] \times$ $[e, g]$, so the formula of the correction term for the to triple integral I using MSuM becomes:

$$
\begin{aligned}
& E_{M S u M}(t)=(b-a)(d-c)(f-e) \\
& \left(\frac{\mathrm{t}^{2}}{6} \frac{\partial^{2} h\left(\bar{\varpi}_{1}, \bar{\theta}_{1}, \bar{\vartheta}_{1}\right)}{\partial \alpha^{2}}-\right. \\
& \frac{7 t^{4}}{360} \frac{\partial^{4} h\left(\overline{\varpi_{2}}, \overline{\theta_{2}}, \overline{\vartheta_{2}}\right)}{\partial \alpha^{4}} \\
& \left.+\frac{31 t^{6}}{15120} \frac{\partial{ }^{6} h\left(\overline{\varpi_{3}}, \overline{\theta_{3}}, \overline{\vartheta_{3}}\right)}{\partial \alpha^{6}}-\cdots\right) \\
& +(b-a)(d-c)(f-e) \\
& \left(\frac{t^{2}}{24} \frac{\partial^{2} h\left(\overline{\overline{\bar{\sigma}_{1}}}, \overline{\bar{\theta}_{1}}, \overline{\overline{\vartheta_{1}}}\right)}{\partial \kappa^{2}}\right. \\
& \left.-\frac{5 t^{4}}{1440} \frac{\partial^{4} h\left(\overline{\overline{\bar{\sigma}_{2}}}, \overline{\overline{\theta_{2}}}, \overline{\overline{\vartheta_{2}}}\right)}{\partial \kappa^{4}}+\frac{61 t^{6}}{60480} \frac{\partial^{6} h\left(\overline{\overline{\bar{\sigma}_{3}}}, \overline{\overline{\theta_{3}}}, \overline{\overline{\vartheta_{3}}}\right)}{\partial \kappa^{6}}-\ldots\right) \\
& +(b-a)(d-c)(f-e)\left(\frac{t^{2}}{6} \frac{\partial^{2} h\left(\overline{\left.\overline{\overline{\varpi_{1}}}, \overline{\overline{\theta_{1}}}, \overline{\overline{\vartheta_{1}}}\right)}\right.}{\partial \rho^{2}}-\right. \\
& \left.\frac{7 t^{4}}{360} \frac{\partial^{4} h\left(\overline{\overline{\overline{\omega_{2}}}}, \overline{\overline{\theta_{2}}}, \overline{\overline{\vartheta_{2}}}\right)}{\partial \rho^{4}}+\frac{31 t^{6}}{15120} \frac{\partial^{6} h\left(\overline{\overline{\bar{\sigma}_{3}}}, \overline{\overline{\theta_{3}}}, \overline{\overline{\vartheta_{1}}}\right)}{\partial \rho^{6}}-\cdots\right)
\end{aligned}
$$

$$
\begin{aligned}
& E_{\text {MSuM }}(t)=(b-a)(d-c)(f-e) \\
& \frac{\mathrm{t}^{2}}{6}\left(\frac{\partial^{2} h\left(\overline{\varpi_{1}}, \overline{\theta_{1}}, \overline{\vartheta_{1}}\right)}{\partial \alpha^{2}}+\right. \\
& \left.+\frac{1}{4} \frac{\partial^{2} h\left(\overline{\overline{\bar{\varpi}_{1}}}, \overline{\overline{\theta_{1}}}, \overline{\overline{\vartheta_{1}}}\right)}{\partial \kappa^{2}}+\frac{\partial^{2} h\left(\overline{\overline{\overline{\bar{\varpi}_{1}}}}, \overline{\overline{\theta_{1}}}, \overline{\overline{\vartheta_{1}}}\right)}{\partial \rho^{2}}\right)+ \\
& +(b-a)(d-c)(f-e) \frac{t^{4}}{360}\left[\left(-7 \frac{\partial^{4} h\left(\overline{\varpi_{2}}, \overline{\theta_{2}}, \overline{\vartheta_{2}}\right)}{\partial \alpha^{4}}-\right.\right. \\
& \left.-\frac{5}{4} \frac{\partial^{4} h\left(\overline{\overline{\bar{\varpi}_{2}}}, \overline{\overline{\theta_{2}}}, \overline{\overline{\vartheta_{2}}}\right)}{\partial \kappa^{4}}-7 \frac{\partial^{4} h\left(\overline{\overline{\overline{\bar{\sigma}_{2}}}}, \overline{\overline{\theta_{2}}}, \overline{\overline{\vartheta_{2}}}\right)}{\partial \rho^{4}}\right)+ \\
& (b-a)(d-c)(f-e) \frac{t^{6}}{15120}\left(31 \frac{\partial^{6} h\left(\overline{\bar{\varpi}_{3}}, \overline{\theta_{3}}, \overline{\vartheta_{3}}\right)}{\partial \alpha^{6}}\right. \\
& \left.+\frac{61}{4} \frac{\partial^{6} h\left(\overline{\overline{\bar{\varpi}_{3}}}, \overline{\overline{\bar{\theta}_{3}}}, \overline{\overline{\vartheta_{3}}}\right)}{\partial \kappa^{6}}+31 \frac{\partial^{6} h\left(\overline{\overline{\overline{\bar{\varpi}_{3}}}}, \overline{\overline{\bar{\theta}_{3}}}, \overline{\overline{\vartheta_{1}}}\right)}{\partial \rho^{6}}\right)+\cdots
\end{aligned}
$$

where, $\cdots,\left(\overline{\overline{\varpi_{r}}}, \overline{\overline{\theta_{r}}}, \overline{\overline{\vartheta_{r}}}\right),\left(\overline{\overline{\varpi_{r}}}, \overline{\theta_{r}}, \overline{\overline{\vartheta_{r}}}\right),\left(\overline{\varpi_{r}}, \overline{\theta_{r}}, \overline{\vartheta_{r}}\right) \in[a, b] \times$ $[c, d] \times[e, f], r=1,2,3, \ldots$

Thus, if the integrand was continuous function and its partial derivatives exists in each point from the integral $[a, b] \times[c, d] \times[e, g]$ we can write the error formula to the above method follows:

$$
I-\operatorname{MSuM}(t)=\gamma_{1} t^{2}+\gamma_{2} t^{4}+\gamma_{3} t^{6}+\cdots
$$

where, $\gamma_{1}, \gamma_{2}, \gamma_{3}, \cdots$ are invariable depends on the partial derivatives for the function in the integral region.

\subsection{Numerical method SuMSu}

Theorem(2): If $\mathrm{h}(\alpha, \kappa, \rho)$ continuous and differentiable function at any point of the region $D=[a, b] \times[c, d] \times[e, f]$ then the approximated value of integral $I=$ $\iint_{D} \int h(\alpha, \kappa, \rho) d \alpha d \kappa d \rho$ can be determined by:

$$
\begin{aligned}
& \text { SuMSu }=\frac{t^{3}}{16} \sum_{j=1}^{w}\left[h\left(a, \kappa_{j}, e\right)+h\left(a, \kappa_{j}, f\right)\right. \\
& +h\left(b, \kappa_{j}, e\right)+h\left(b, \kappa_{j}, f\right)+ \\
& 2\left(h\left(a, \kappa_{j}, e+(w-0.5) t\right)\right. \\
& +h\left(b, \kappa_{j}, e+(w-0.5) t\right) \\
& +h\left(a+(w-0.5) t, \kappa_{j}, e\right) \\
& +h\left(a+(w-0.5) t, \kappa_{j}, f\right) \\
& +2 h\left(a+(w-0.5) t, \kappa_{j}, e\right.
\end{aligned}
$$




$$
\begin{aligned}
& +(w-0.5) t))+2 \sum_{\ell=1}^{w-1}\left(h\left(a, \kappa_{j}, e+(\ell-0.5) t\right)\right. \\
& +h\left(b, \kappa_{j}, e+(\ell-0.5) t\right)+ \\
& +2 h\left(a+(w-0.5) t, \kappa_{j}, e\right. \\
& +(\ell-0.5) t)+h\left(a, \kappa_{j}, e+\ell t\right) \\
& +h\left(b, \kappa_{j}, e+\ell t\right)+2 h(a+(w-0.5) \\
& \left.\left.t, \kappa_{j}, e+\ell t\right)\right)+2 \sum_{i=1}^{w-1}\left(h\left(a+(i-0.5) t, \kappa_{j}, e\right)\right. \\
& +h\left(a+(i-0.5) t, \kappa_{j}, f\right)+ \\
& +h\left(a+i t, \kappa_{j}, e\right)+h\left(a+i t, \kappa_{j}, f\right) \\
& +2\left(h \left(a+(i-0.5) t, \kappa_{j}, e+\right.\right. \\
& \left.+(w-0.5) t+h\left(a+i t, \kappa_{j}, e+(w-0.5) t\right)\right)+ \\
& +2 \sum_{\ell=1}^{w-1}\left(h\left(a+(i-0.5) t, \kappa_{j}, e+(\ell-0.5) t\right)+\right. \\
& +h\left(a+(i-0.5) t, \kappa_{j}, e+\ell t\right)+ \\
& \left.+h\left(a+i t, \kappa_{j}, e+(\ell-0.5) t\right)\right] \\
& \left.\left.+h\left(a+i t, \kappa_{j}, e+\ell t\right)\right)\right) \\
& \kappa_{j}=c+(j-0.5) t, \\
& j=1,2, \ldots, w
\end{aligned}
$$

and the formula of correction terms is

$$
I-\operatorname{SuMSu}(t)=\psi_{1} t^{2}+\psi_{2} t^{4}+\psi_{3} t^{6}+\cdots
$$

where, $\psi_{1}, \psi_{2}, \psi_{3}, \ldots$ are invariable.

Proof: Rewrite the integral $I$ as:

$$
\begin{aligned}
& I=\int_{e}^{f} \int_{c}^{d} \int_{a}^{b} h(\alpha, \kappa, \rho) d \alpha d \kappa d \rho \\
& =\operatorname{SuMSu}(t)+\delta_{\text {SuMSu }}(t)
\end{aligned}
$$

where, $\operatorname{SuMSu}(t)$ : is the approximation value of integral by using suggested method on the first dimension X.

Mid-point method on the second dimension $\mathrm{Y}$ with suggested method on the third dimension $Z, \delta_{\text {SuMSu }}(t)$ are the correction terms that would be added to the values of $\operatorname{SuMSu}(\mathrm{t})$, and

$$
t=\left(\frac{(b-a)}{w}\right)=\left(\frac{(d-c)}{w_{1}}\right)=\left(\frac{(f-e)}{w_{2}}\right)
$$

By following the same procedure in the previous proof, we get:

$$
I-\operatorname{SuMSu}(t)=\psi_{1} t^{2}+\psi_{2} t^{4}+\psi_{3} t^{6}+\cdots
$$

Such that $\psi_{1}, \psi_{2}, \psi_{3}, \cdots$ are invariable depends on the partial derivatives for the function in the integration region.

\section{CALCULATING THE TRIPLE INTEGRALS USING R(MSUM) AND R(SUMSU) METHODS}

To assess the triple integrals numerically by using the methods $R O(M S u M)$ and $R O(S u M S u)$, first must compute the approximated value of the integral $I$ where $w=1$ using $M S u M$ which is:

$$
\begin{aligned}
& \int_{e}^{f} \int_{c}^{b} \int_{a}^{b} h(\alpha, \kappa, \rho) d \alpha d \kappa d \rho \\
& =\frac{t^{3}}{4}[h(a+0.5 t, c, e+0.5 t) \\
& +h(a+0.5 t, d, e+0.5 t) \\
& +2 h(a+0.5 t, c+0.5 t, e+0.5 t)]
\end{aligned}
$$

then fixed this value in our tables.

Now, to find another approximated value put $w=2$, then:

$$
\begin{aligned}
& \int_{e}^{f} \int_{c}^{d} \int_{a}^{b} h(\alpha, \kappa, \rho) d \alpha d \kappa d \rho \\
& =\frac{t^{3}}{4} \sum_{\ell=1}^{2} \sum_{i=1}^{2}\left[h\left(\alpha_{i}, c, \rho_{\ell}\right)+h\left(\alpha_{i}, d, \rho_{\ell}\right)\right. \\
& +2 h\left(\alpha_{i}, c+(w-0.5) t, \rho_{\ell}\right) \\
& \left.+2\left(h\left(\alpha_{i}, c+0.5 t, \rho_{\ell}\right)+h\left(\alpha_{i}, c+t, \rho_{\ell}\right)\right)\right]
\end{aligned}
$$

$w$ is the number of subintervals that divides the integration interval and the base $\mathrm{y}$ is $w=2^{k}, k=0,1,2, \ldots$

Again, fixed this value in our tables as the approximate value for the triple integral I. We can improve these two approximate values using Romberg accelerating. We continue to apply $M S u M$ rule for the other value $w>2$.

Note that: We used the Matlab language to write programs for this search to obtain the blog results in tables for the triple integrations below

\section{SOME EXAMPLES AND RESULTS}

Example (1): The integral

$\int_{\frac{\pi}{4}}^{\frac{\pi}{2}} \int_{\frac{\pi}{4}}^{\frac{\pi}{2}} \int_{\frac{\pi}{4}}^{\frac{\pi}{2}} \alpha \sin (3.2 \kappa-1.5 \rho) d \alpha d \kappa d \rho$ which its analytical value is equal to 0.369989494366 (approximated to 12 decimal digits) which is continuous integrand for every point $(\alpha, \kappa, \rho) \in\left[\frac{\pi}{2}, \frac{\pi}{4}\right] \times\left[\frac{\pi}{2}, \frac{\pi}{4}\right] \times\left[\frac{\pi}{2}, \frac{\pi}{4}\right] \quad$. Consequently, the correction terms of this integral are similar to the two formulas (29) and (31). When we applied $R O$ (MSuM) and $R O($ SuMSu) are respectively, we got the results that are recorded in Tables 1 and 2 some details of these two tables are:

1. Table 1: when $w=16$. By utilizing MSuM rule (i.e. equal to the analytical value of Romberg acceleration), the approximated value is correct of the three decimal places. note that the time taken by the Matlab program to calculate was (2,675 seconds). compared with Eghaar [3] when she got a correct value for nine correct decimal digits at $w=16$ with the same number of partial periods, while Dheyaa [2] got a correct value for six correct decimal digits at $\mathrm{w}=16$.

2. Table 2: when $w=32$. By utilizing $S u M S u$ rule (i.e. equal 
to analytical value with Romberg acceleration), the approximated value is correct for one decimal places. note that the time taken by the Matlab program to calculate was $(13,057$ seconds). compared with Eghaar [3] when she got a correct value for eleven correct decimal digits at $w=32$ with the same number of partial periods, while Dheyaa [2] got a correct value for seven correct decimal digits at $w=32$. Therefore, method of $R O(M S u M)$ is the best in accuracy and quickness of approach.

Example (2): The analytical value of the integral $\int_{3}^{4} \int_{2}^{3} \int_{1}^{2} \sqrt{\kappa+\rho} \ln (\alpha+\kappa+z) d \alpha d \kappa d \rho$ is equal to 4.931709266345 (approximated to 12 decimal digits) which is continuous integrand for every point $(\alpha, \kappa, \rho) \in[1,2] \times$ $[2,3] \times[3,4]$. Therefore, the results of this integral are similar to the two formulas (29) and (31). When we applied $R O(M S u M)$ and $R O(S u M S u)$ are respectively, we got the results that are listed in Tables 3 and 4 some details of these two tables are:-

1. Table 3: when $w=16$.

By uitilzing MSuM rule (i.e. equal to analytical value with Romberg acceleration), the approximated value is correct for four decimal places. note that the time taken by the Matlab program to calculate was (8.764 seconds). compared with Eghaar [3] when she got a correct value for eleven correct decimal digits at $w=16$ with the same number of partial periods, while Dheyaa [2] got a correct value for also nine correct decimal digits at $w=16$.

2. Table 4: when $w=8$. By utilizing SuMSu rule (i.e. equal to analytical value with Romberg acceleration), the approximated value is correct for five decimal places. note that the time taken by the Matlab program to calculate was (1.559 seconds) compared with Eghaar [3] when she got a correct value for ten correct decimal digits at $w=8$, while Dheyaa [2] got a correct value for eight correct decimal digits at $w=8$.

Table 1. $\int_{\frac{\pi}{4}}^{\frac{\pi}{2}} \int_{\frac{\pi}{4}}^{\frac{\pi}{2}} \int_{\frac{\pi}{4}}^{\frac{\pi}{2}} \alpha \sin (3.2 \kappa-1.5 \rho) d \alpha d \kappa d \rho R O(M S u M)$

\begin{tabular}{cccccc}
\hline $\mathbf{w}$ & $\mathbf{M S u M}$ & $\mathbf{R = 2}$ & $\mathbf{R}=\mathbf{4}$ & $\mathbf{R = 6}$ & $\mathbf{R = 8}$ \\
\hline 1 & 0.339250421995 & & & & \\
2 & 0.362961251612 & 0.370864861485 & & & \\
4 & 0.368271055916 & 0.370040990684 & 0.369986065964 & & \\
8 & 0.369562262914 & 0.369992665246 & 0.369989443550 & 0.369989497163 & \\
16 & 0.369882834587 & 0.369989691812 & 0.369989493583 & 0.369989494377 & 0.369989494366 \\
$\mathbf{w}$ & $\mathbf{M M M}$ & $\mathbf{R = 2}$ & $\mathbf{R}=\mathbf{4}$ & $\mathbf{R}=\mathbf{6}$ & $\mathbf{R = 8}$ \\
16 & 0.370453827346 & 0.369987997697 & 0.369989510933 & 0.369989493654 & 0.369989494504 \\
$\mathbf{w}$ & & & $\mathbf{R M R S ( R S )}$ & & \\
16 & & & 0.369989132488 & & \\
\hline
\end{tabular}

Table 2. $\int_{\frac{\pi}{4}}^{\frac{\pi}{2}} \int_{\frac{\pi}{4}}^{\frac{\pi}{2}} \int_{\frac{\pi}{4}}^{\frac{\pi}{2}} \alpha \sin (3.2 \kappa-1.5 \rho) d \alpha d \kappa d \rho \backslash R O(S u M S u)$

\begin{tabular}{|c|c|c|c|c|c|c|}
\hline $\mathbf{w}$ & SuMSu & $\mathbf{R}=\mathbf{2}$ & $R=4$ & $R=6$ & $\mathbf{R}=\mathbf{8}$ & $\mathbf{R}=\mathbf{1 0}$ \\
\hline 1 & 0.474651468633 & & & & & \\
\hline 2 & 0.392640597516 & 0.365303640477 & & & & \\
\hline 4 & 0.375466383269 & 0.369741645187 & 0.370037512168 & & & \\
\hline 8 & 0.371347542666 & 0.369974595798 & 0.369990125839 & 0.369989373675 & & \\
\hline 16 & 0.370328314734 & 0.369988572090 & 0.369989503843 & 0.369989493970 & 0.369989494442 & \\
\hline 32 & 0.370074156330 & 0.369989436862 & 0.369989494513 & 0.369989494365 & 0.369989494367 & 0.369989494366 \\
\hline $\mathbf{w}$ & МMM & $R=2$ & $R=4$ & $R=6$ & $R=8$ & $R=10$ \\
\hline 32 & 0.370105507635 & 0.369989401065 & 0.369989494623 & 0.369989494354 & 0.369989494363 & 0.369989494367 \\
\hline $\mathbf{w}$ & \multicolumn{6}{|c|}{ RMRS(RS) } \\
\hline 32 & \multicolumn{6}{|c|}{0.369989476187} \\
\hline
\end{tabular}

Table 3. $\int_{3}^{4} \int_{2}^{3} \int_{1}^{2} \sqrt{\kappa+\rho} \ln (\alpha+\kappa+\rho) d \alpha d \kappa d \rho \backslash R O(M S u M)$

\begin{tabular}{cccccc}
\hline $\mathbf{w}$ & $\mathbf{M S u M}$ & $\mathbf{R = 2}$ & $\mathbf{R = 4}$ & $\mathbf{R = 6}$ & $\mathbf{R = 8}$ \\
\hline 1 & 4.934022219352 & & & & \\
2 & 4.932289001613 & 4.931711262367 & & & \\
4 & 4.931854294754 & 4.931709392467 & 4.931709267807 & & \\
8 & 4.931745529376 & 4.931709274250 & 4.931709266369 & 4.931709266346 & \\
16 & 4.931718332474 & 4.931709266840 & 4.931709266346 & 4.931709266345 & 4.931709266345 \\
$\mathbf{w}$ & $\mathbf{M M M}$ & $\mathbf{R = 2}$ & $\mathbf{R = 4}$ & $\mathbf{R}=\mathbf{6}$ & $\mathbf{R = 8}$ \\
16 & 4.931724072493 & 4.931709267301 & 4.931709266121 & 4.931709266334 & 4.931709266342 \\
$\mathbf{w}$ & & & $\mathbf{R M R S ( R S )}$ & & \\
16 & & & 4.931709266589 & & \\
\hline
\end{tabular}


Therefore, method of $\mathrm{RO}(\mathrm{SuMSu})$ is the best in speed of approach.

Example (3): The integral $I=$ $\int_{3.5}^{4} \int_{2.5}^{3} \int_{1.5}^{2} \frac{\kappa}{\sqrt{(1+\alpha \rho)^{3}}} d \alpha d \kappa d \rho$ which it's analytical value is equal to 0.062596066956 (approximated to 12 decimal digits) which is continuous integrand for all point $(\alpha, \kappa, \rho) \in$ $[1.5,2] \times[2.5,3] \times[3.5,4]$. By applying, the obtained results are shown in Tables 5 and 6 .

1. Table 5: when $w=16$. By utilizing MSuM rule, the approximated value is correct for five decimal places. note that the time taken by the Matlab program to calculate was $(2,861$ seconds).

compared with Eghaar [3] when she got a correct value for eleven correct decimal digits at $w=16$, while Dheyaa [2] got a correct value for ten correct decimal digits at $w=16$.

2. Table 6: when $w=8$. By utilizing SuMSu rule, the approximated value is correct for three decimal places. note that the time taken by the Matlab program to calculate was (2,062 seconds).

compared with Eghaar [3] when she got a correct value for ten correct decimal digits at $w=16$, while Dheyaa [2] got a correct value for eight correct decimal digits at $w=16$.
Hence, method of $R O(S u M S u)$ is the best in speed of approach.

Example (4): - The integral $I=\int_{2}^{3} \int_{2}^{3} \int_{2}^{3}(\alpha \kappa \rho)^{\frac{1}{\kappa \rho}} d \alpha d \kappa d \rho$ which analytical value is unknown. It has a continuous integrand for each point $(\alpha, \kappa, \rho) \in[2,3] \times[2,3] \times[2,3]$.

Thus, the formula for the correction of this integral is identical to the two formulas (29) and (31) respectively. When we applied $R O(M S u M)$ and $R O(S u M S u)$ are respectively, we found the results that are listed in Tables 7 and 8 some details of these two tables are:

where the value is constant (for twelve decimal times) for the last two columns when $w=16$ and for the last three columns when $w=32$ for the two methods that are mentioned above, thus this indicates which is the value of the above integral is rounded to twelve decimal times which are 1.561064101183. Note that the time it took for the two programs the Matlab to calculate were ( 8.587 seconds) and (14,193 seconds) using two methods $R O(M S u M)$ and $R O(S u M S u)$ are respectively. compared with Eghaar in [3] when she noticed that the value is constant for the last three columns when at $w=32$, while Dheyaa in [2] got a correct value for ten correct decimal digits at $w=32$.

Table 4. $\int_{3}^{4} \int_{2}^{3} \int_{1}^{2} \sqrt{\kappa+\rho} \ln (\alpha+\kappa+\rho) d \alpha d \kappa d \rho \backslash R O($ SuMSu $)$

\begin{tabular}{ccccc}
\hline $\mathbf{w}$ & SuMSu & $\mathbf{R = 2}$ & $\mathbf{R}=\mathbf{4}$ & $\mathbf{R = 6}$ \\
\hline 1 & 4.931284952484 & & & \\
2 & 4.931602920229 & 4.931708909478 & & \\
4 & 4.931682663012 & 4.931709243940 & 4.931709266237 & \\
8 & 4.931702614461 & 4.931709264943 & 4.931709266344 & 4.931709266345 \\
$\mathbf{w}$ & $\mathbf{M M M}$ & $\mathbf{R = 2}$ & $\mathbf{R = 4}$ & $\mathbf{R = 6}$ \\
8 & 4.931768488068 & 4.931709281623 & 4.931709266406 & 4.931709266337 \\
$\mathbf{w}$ & & $\mathbf{R M R S}(\mathbf{R S})$ & \\
8 & & \multicolumn{2}{c}{4.931709267767} & \\
\hline
\end{tabular}

Table 5. $I=\int_{3.5}^{4} \int_{2.5}^{3} \int_{1.5}^{2} \frac{\kappa}{\sqrt{(1+\alpha \rho)^{3}}} d \alpha d \kappa d \rho \backslash R O(M S u M)$

\begin{tabular}{cccccc}
\hline $\mathbf{w}$ & $\mathbf{M S u M}$ & $\mathbf{R = 2}$ & $\mathbf{R}=\mathbf{4}$ & $\mathbf{R}=\mathbf{6}$ & $\mathbf{R = 8}$ \\
\hline 1 & 0.061983471074 & & & & \\
2 & 0.062439740794 & 0.062591830700 & & & \\
4 & 0.062556775941 & 0.062595787656 & 0.062596051454 & & \\
8 & 0.062586230922 & 0.062596049249 & 0.062596066689 & 0.062596066931 & \\
16 & 0.062593607114 & 0.062596065845 & 0.062596066951 & 0.062596066955 & 0.062596066956 \\
$\mathbf{w}$ & $\mathbf{M M M}$ & $\mathbf{R = \mathbf { 2 }}$ & $\mathbf{R = 4}$ & $\mathbf{R}=\mathbf{6}$ & $\mathbf{R}=\mathbf{8}$ \\
16 & 0.062594718332 & 0.062596174934 & 0.062596076772 & 0.062596066943 & 0.062596066954 \\
$\mathbf{w}$ & & & $\mathbf{R M R S ( R S )}$ & & \\
16 & & & 0.062596066923 & & \\
\hline
\end{tabular}

Table 6. $I=\int_{3.5}^{4} \int_{2.5}^{3} \int_{1.5}^{2} \frac{\kappa}{\sqrt{(1+\alpha \rho)^{3}}} d \alpha d \kappa d \rho \backslash R O($ SuMSu $)$

\begin{tabular}{ccccc}
\hline $\mathbf{w}$ & SuMSu & $\mathbf{R = 2}$ & $\mathbf{R = 4}$ & $\mathbf{R = 6}$ \\
\hline 1 & 0.062909622398 & & & \\
2 & 0.062674706174 & 0.062596400766 & & \\
4 & 0.062615742608 & 0.062596088086 & 0.062596067241 & \\
8 & 0.062600986862 & 0.062596068280 & 0.062596066960 & 0.062596066956 \\
$\mathbf{w}$ & $\mathbf{M M M}$ & $\mathbf{R = 2}$ & $\mathbf{R}=\mathbf{4}$ & $\mathbf{R}=\mathbf{6}$ \\
8 & 0.062595628223 & 0.062596074934 & 0.062596068881 & 0.062596066944 \\
$\mathbf{w}$ & & \multicolumn{2}{c}{$\mathbf{R M R S ( R S )}$} & \\
8 & & \multicolumn{2}{c}{0.062596067877} & \\
\hline \multicolumn{4}{c}{} \\
\hline
\end{tabular}


Table 7. $I=\int_{2}^{3} \int_{2}^{3} \int_{2}^{3}(\alpha \kappa \rho)^{\frac{1}{\kappa \rho}} d \alpha d \kappa d \rho \backslash R O(M S u M)$

\begin{tabular}{|c|c|c|c|c|c|c|}
\hline $\mathbf{w}$ & MSuM & $\mathbf{R}=\mathbf{2}$ & $R=4$ & $R=6$ & $\mathbf{R}=\mathbf{8}$ & $\mathbf{R}=\mathbf{1 0}$ \\
\hline 1 & 1.560070541592 & & & & & \\
\hline 2 & 1.560789704179 & 1.561029425041 & & & & \\
\hline 4 & 1.560993720384 & 1.561061725786 & 1.561063879169 & & & \\
\hline 8 & 1.561046391950 & 1.561063949139 & 1.561064097362 & 1.561064100825 & & \\
\hline 16 & 1.561059666705 & 1.561064091623 & 1.561064101122 & 1.561064101183 & 1.561064101183 & \\
\hline 32 & 1.561062992115 & 1.561064100585 & 1.561064101182 & 1.561064101183 & 1.561064101183 & 1.561064101183 \\
\hline $\mathbf{w}$ & МMM & $\mathbf{R}=\mathbf{2}$ & $R=4$ & $R=6$ & $\mathbf{R}=\mathbf{8}$ & $\mathbf{R}=\mathbf{1 0}$ \\
\hline 16 & 1.561028976357 & 1.561064078011 & 1.561064101092 & 1.561064101184 & 1.561064101184 & \\
\hline 32 & 1.561055318889 & 1.561064099733 & 1.561064101181 & 1.561064101183 & 1.561064101183 & 1.561064101183 \\
\hline $\mathrm{w}$ & \multicolumn{6}{|c|}{ RMRS $(\mathbf{R S})$} \\
\hline 32 & \multicolumn{6}{|c|}{1.561064101166} \\
\hline
\end{tabular}

Table 8. $I=\int_{2}^{3} \int_{2}^{3} \int_{2}^{3}(\alpha \kappa \rho)^{\frac{1}{\kappa \rho}} d \alpha d \kappa d \rho \backslash R O(S u M S u)$

\begin{tabular}{ccccccc}
\hline $\mathbf{w}$ & SuMSu & $\mathbf{R = 2}$ & $\mathbf{R = 4}$ & $\mathbf{R = 6}$ & $\mathbf{R = 8}$ & $\mathbf{R = 1 0}$ \\
\hline 1 & 1.55789959568 & & & & & \\
2 & 1.56023703284 & 1.56101611190 & & & & \\
4 & 1.56085482584 & 1.56106085684 & 1.56106381984 & & & \\
8 & 1.56101139939 & 1.56106392390 & 1.56106406171 & 1.561064106812 & & \\
16 & 1.56105096028 & 1.56106408058 & 1.56106411103 & 1.56106401183 & 1.561064111833 & \\
32 & 1.56106089278 & 1.56106410361 & 1.56106411182 & 1.56106411183 & 1.56106410183 & 1.561064101833 \\
\hline
\end{tabular}

Table 9. $\int_{2}^{3} \int_{2}^{3} \int_{2}^{3} \frac{\rho \sinh \left(\frac{\pi \alpha}{2}\right)+\cosh \left(\frac{\pi \kappa}{2}\right)}{\sqrt{\alpha^{4}+\kappa^{4}+\rho^{4}}} d \alpha d \kappa d \rho \backslash R O(M S u M)$

\begin{tabular}{|c|c|c|c|c|c|c|c|}
\hline $\begin{array}{l}\mathbf{w} \\
1\end{array}$ & $\begin{array}{c}\text { MSuM } \\
8.394612480458\end{array}$ & $\mathbf{R}=\mathbf{2}$ & $R=4$ & $R=6$ & $\mathbf{R}=\mathbf{8}$ & $R=10$ & $R=12$ \\
\hline 2 & 8.549693284606 & 8.601386885988 & & & & & \\
\hline 4 & 8.591441476046 & 8.605357539860 & 8.605622250118 & & & & \\
\hline 8 & 8.602067383103 & 8.605609352122 & 8.605626139606 & 8.605626201344 & & & \\
\hline 16 & 8.604735717631 & 8.605625162474 & 8.605626216497 & 8.605626217718 & 8.605626217782 & & \\
\hline 32 & 8.605403543269 & 8.605626151815 & 8.605626217772 & 8.605626217792 & 8.605626217792 & 8.605626217792 & \\
\hline 64 & 8.605570546069 & 8.605626213668 & 8.605626217792 & 8.605626217792 & 8.605626217792 & 8.605626217792 & 8.605626217792 \\
\hline $\mathbf{w}$ & МMM & $\mathbf{R}=\mathbf{2}$ & $R=4$ & $R=6$ & $\mathbf{R}=\mathbf{8}$ & $\mathbf{R}=\mathbf{1 0}$ & $R=12$ \\
\hline 32 & 8.605213089643 & 8.605626135507 & 8.605626217747 & 8.605626217791 & 8.605626217791 & 8.605626217792 & \\
\hline 64 & 8.605522931897 & 8.605626212648 & 8.605626217791 & $\begin{array}{c}8.605626217792 \\
\text { RMRS(RS) }\end{array}$ & 8.605626217792 & 8.605626217792 & 8.605626217792 \\
\hline 64 & & & & 8.605626217744 & & & \\
\hline
\end{tabular}

Table 10. $\int_{2}^{3} \int_{2}^{3} \int_{2}^{3} \frac{\rho \sinh \left(\frac{\pi \alpha}{2}\right)+\cosh \left(\frac{\pi \kappa}{2}\right)}{\sqrt{\alpha^{4}+\kappa^{4}+\rho^{4}}} d \alpha d \kappa d \rho \backslash R O(S u M S u)$

\begin{tabular}{cccccccc}
\hline $\mathbf{w}$ & SuMSu & $\mathbf{R = 2}$ & $\mathbf{R = 4}$ & $\mathbf{R = 6}$ & $\mathbf{R = 8}$ & $\mathbf{R = 1 0}$ & $\mathbf{R = 1 2}$ \\
\hline 1 & 8.628057466238 & & & & & & \\
2 & 8.610134912884 & 8.604160728433 & & & & & \\
4 & 8.606681791962 & 8.605530751655 & 8.605622086536 & & & & \\
8 & 8.605885593887 & 8.605620194529 & 8.605626157387 & 8.605626222004 & & & \\
16 & 8.605690778813 & 8.605625840454 & 8.605626216849 & 8.605626217793 & 8.6056262177777 & & \\
32 & 8.605642340349 & 8.605626194195 & 8.605626217777 & 8.605626217792 & 8.605626217792 & 8.605626217792 & \\
64 & 8.605630247325 & 8.605626216317 & 8.605626217792 & 8.605626217792 & 8.605626217792 & 8.605626217792 & 8.605626217792 \\
\hline
\end{tabular}

We obtained from Tables 7 and 8 the same results, so we showed the comparison in Table 7 only.

\section{Example}

(5):

The integral $\int_{2}^{3} \int_{2}^{3} \int_{2}^{3} \frac{\rho \sinh \left(\frac{\pi \alpha}{2}\right)+\cosh \left(\frac{\pi \kappa}{2}\right)}{\sqrt{\alpha^{4}+\kappa^{4}+\rho^{4}}} d \alpha d \kappa d \rho$ which analytical value is unknown. Also, it has a continuous integrand for each point $(\alpha, \kappa, \rho) \in[2,3] \times[2,3] \times[2,3]$. The obtained results through applied $R O(M S u M)$ and $R O(S u M S u)$ are shown in Tables 9 and 10, respectively. Some details of these two tables are:

The value is constant (for twelve decimal times) for the last three columns when $w=32$ and for the last five columns when 
$w=64$ for the two methods that are mentioned above, thus this indicates that the value of the above integral is rounded to twelve decimal times which are 8.605626217792. Note that the time it took for the two programs the Matlab to calculate were (10.319seconds) and $(14,193$ seconds) using two methods $R O(M S u M)$ and $R O(S u M S u)$ are respectively. compared with Eghaar [3] she got the same results as we did, while Dheyaa [2] got a correct value for ten correct decimal digits at $w=64$.

We obtained from Tables 9 and 10 the same results, so we showed the comparison in Table 9 only.

\section{CONCLUSION}

The results in this investigation display that the computation of the approximate values for the triple integrals that continuous integrands using two complex methods $R O(M S u M)$ and $R O(S u M S u)$ when the number of divisions are equals on these three dimensions which gives exact values (for several decimal times) with Romberg acceleration compared with exact values of integral using number from sub-intervals. In addition, we obtained accuracy which is equal to twelve decimal times with sub-intervals between (8-64) for both previous methods.

The methods $R O(M S u M)$ and $R O(S u M S u)$ have several applications, including computing numerically triple integrals that go into finding volumes, intermediate centers, and inertia torque of volumes, also find blocks of variable density such as a thin wire or a thin plate of metal [1].

Advantages of the two methods ( $R O(M S u M)$ and $R O(S u M S u)$ ): Finding the value of integrals whose values cannot be found analytically and using it to find the values of complicated integrals whose values are analytically possible, but laborious, because the function is complex and it takes a long time to find the value of the integral.

The disadvantages of the two methods ( $R O(M S u M)$ and $R O(S u M S u))$ : They cannot be used to calculate the indefinite integral.

The method used in this article can be generalized and applied to quadruple integration.

\section{REFERENCES}

[1] Ayres, F., Mendelson, E. (1999). Schaum's Outline of Calculus. McGraw-Hill Book-Company.

[2] Dheyaa, A.M. (2009). Some numerical methods for calculating single, double, and triple integrals using Matlab language. Doctoral Dissertation, University of Kufa.

[3] Eghaar, B. (2010). Some numerical methods for calculating double and triple integrals. Doctoral Dissertation, MSc Dissertation, University of Kufa.

[4] Muosa, S.M., Salman, M.R., Jubair, H.A. (2017). Aitken's Acceleration Method with Mid-point rule on the dimension of exterior and Simpson's rule on the two dimensions of middle and exterior to Evaluation Triple Integrals Numerically. Journal of Kerbala University, 15(2): 206-212.

[5] Mohammed, A.H., Aljassas, S.M., Mohammed, W. (2013). Derivation of numerically method for evaluating triple integrals with continuous integrands and form of error (Correction terms). Journal of Kerbala University, 11(4): 67-76.

[6] Shubar, A.W.K. (2014). Derivation of numerical methods of newton-coates formulas for the calculation of specific triple integrations and improvement of results using experimental methods. Doctoral Dissertation, University of Kufa.

[7] Naser, R.H. (2018). Using numerical methods to solve continuous triple integrals. International Journal of Pure and Applied Mathematics, 119(10): 349-358.

[8] Jayan, S., Nagaraja, K.V. (2015). A general and effective numerical integration method to evaluate triple integrals using generalized Gaussian quadrature. Procedia Engineering, 127: 1041-1047. https://doi.org/10.1016/j.proeng.2015.11.457

[9] AL-Gasas, S.M.M. (2015). Evaluation of triple integrals with continuous integrands numerically by using method RM (RMM). Journal of Al-Qadisiyah for Computer Science and Mathematics, 7(1): 1-10.

[10] AL-Shimmari, A.A. (2019). Innovative acceleration methods of first kind for Improving the values of integrals numerically. MSc Dissertation, University of Kufa.

[11] Alsharify, F.H., Al-Karamy, N.A.M., Aljassas, S.M. (2018). Extrapolation rule to increase the accuracy of numerical integrals results. Jour of Adv Research in Dynamical \& Control Systems, 10(12): 587-595.

[12] Al-Karamy, N. (2017). New acceleration formula for improvement results of the numerical integration. Journal of Kerbala University, 15(2): 100-113.

[13] AL-Aridhee, S.H.T. (2020). Innovative acceleration methods from second kind for improving results of integrations numerically. MSc Dissertation, University of Kufa.

[14] Fox, L., Hayes, L. (1970). On the definite integration of singular integrands. SIAM Review, 12(3): 449-457. https://doi.org/10.1137/1012084

[15] Mohammed, A.H., Alkiffai, A.N., Khudair, R.A. (2011). Suggested numerical method to evaluate single integrals. Journal of Kerbala University, 9: 201-206.

[16] Ralston, A., Rabinowitz, P. (2001). A first course in numerical analysis. Courier Corporation, pp. 87-94. 